\title{
The gas sensing performances of gas sensors based on the dielectrophoretically manipulated multi-wall carbon nanotubes with various functionalized groups towards $\mathrm{NH}_{3}$
}

\author{
WANG Hai-bo \& FENG Li-li ${ }^{* 1}$,CHEN Hui-ying ${ }^{2}$
}

${ }^{1}$ Key Laboratory of Urban Stormwater System and Water Environment of Ministry of Education, Beijing Climate Change Response Research and Education Center, Beijing University of Civil Engineering and Architecture, Beijing 100044, P. R. China

${ }^{2}$ College of Life and Environmental Sciences, Minzu University of China, Beijing 100081, P. R. China

${ }^{*}$ Corresponding author: Beijing University of Civil Engineering and Architecture, Beijing, 100044, P. R. China;fenglili@bucea.edu.cn

KEYWORD: Dielectrophoretical manipulation; Multi-wall carbon nanotubes; Functional group modification; Gas sensors; Ammonia

ABSTRACT: Gas sensors based on raw multi-wall carbon nanotubes (raw MWNTs) and functionalized multi-wall carbon nanotubes (MWNTs-x) were fabricated on the chromium-silver-gold microelectrode chips by dielectrophoresis process. The positive dielectrophoresis was observed for the particles of carbon nanotubes. The gas sensing performances of the fabricated gas sensors towards different concentrations of $\mathrm{NH}_{3}$ were tested at room temperature. It was found that the response time, recovery time and sensitivity of gas sensors towards $\mathrm{NH}_{3}$ were increased with the increase of $\mathrm{NH}_{3}$ concentrations. We demonstrated that functionalizing MWNTs does not change the adsorption properties of $\mathrm{NH}_{3}$ on MWNTs. In addition, We conclude that the interactions between MWNTs and $\mathrm{NH}_{3}$ such as surface area and small diameter are influential factors for the gas sensing performances of these gas sensors.

\section{Introduction}

Recently, carbon tubes (CNTs) has attracted great interest owing to its unique mechanics, electrical and chemical properties. This makes the CNTs a promising material for a variety of potential applications ${ }^{[1]}$. Previous works have demonstrated that CNTs can detect a wide range of gases at or near room temperature, such as $\mathrm{NO}_{2}, \mathrm{NH}_{3}$, so it is an ideal candidate for sensing material. CNT-based gas sensing utilizes the electrical resistance change of the CNTs due to adsorption of gas molecules as the electricsl readout. Compared to other gas sensors, CNT-based gas sensors have many outstanding properties such as faster response, higher sensitivity, and lower operating temperature $^{[2]}$.

Many research groups use SWNTs for $\mathrm{NO}_{\mathrm{x}}{ }^{[3]}$, Organic vapor ${ }^{[4]}, \mathrm{O}_{3}{ }^{[5]}$ detection and MWNTs for $\mathrm{SF}_{6}{ }^{[6]}, \mathrm{CO}^{[7]}, \mathrm{H}_{2} \mathrm{~S}^{[8,9]}, \mathrm{H}_{2}{ }^{[10,11]}, \mathrm{VOC}^{[12]}$ detection. The recovery time of SWNTs gas sensors are actually long, although the majority of their performances are better than the traditional ones. MWNTs gas sensors receive little attention due to a low sensitivity. It is a challenge to enhance the reduce the response time and recovery time. We attempt to modify MWNTs and manipulate the MWNTs using dielectrophoretic (DEP) in order to solve these problems.

In this study, the gas sensors based on raw MWNTs and MWNTs-x $\left(-\mathrm{OH},-\mathrm{COOH},-\mathrm{NH}_{2}\right)$ were fabricated on the chromium-silver-gold microelectrode chips by DEP. The gas sensing performances of the fabricated gas sensors towards different concentrations of $\mathrm{NH}_{3}$ were tested at room temperature.

\section{Experimental}

All MWNTs from the supplier (Cheaptubes, Brattleboro, USA) were synthesized by chemic-al 
vapour deposition, which have purities of $>99 \%$. Hydroxylated $(\mathrm{OH})$, carboxylated $(\mathrm{COOH})$ and aminoated $\left(\mathrm{NH}_{2}\right)$ MWCNTs were functionalized by plasma treatment and contained $7 \pm 1.5 \mathrm{wt} \%$ functional groups according to X-ray photoelectron spectroscopy (XPS) and titration results provided by the supplier. The porous characteristics of the MWCNTs materials are shown in table 1 .

Table 1 Porous characteristics of the MWNTs materials

\begin{tabular}{llll}
\hline Materials & $S_{\text {BET }}$ & $V_{\text {pore }}$ & $D_{\text {pore }}$ \\
\cline { 2 - 4 } & $\mathrm{m}^{2} \cdot \mathrm{g}^{-1}$ & $\mathrm{~cm}^{3} \cdot \mathrm{g}^{-1}$ & $\mathrm{~nm}$ \\
\hline Raw MWNTs & 224 & 1.86 & 33.2 \\
MWNTs-NH & 206 & 2.12 & 41.2 \\
MWNTs-OH & 205 & 2.31 & 45.3 \\
MWNTs-COOH & 178 & 1.97 & 44.2 \\
\hline
\end{tabular}

All MWNTs were suspended in ultrapure water and ultra-sonicated for $20 \mathrm{~min}$. An finger-protruding microelectrode of thin chrome film was patterned on a quartz glass substrate by vacuum evaporation technique. The electrode had $40 \mu \mathrm{m}$ width and $40 \mu \mathrm{m}$ the minimum clearance.

The MWNTs suspension was dropped into the microelectrode. The DEP trapping of MWNTs to the microelectrode was performed with ac voltage of $2 \mathrm{MHz}$ frequency and $8 \mathrm{~V}$ amplifier (peak to peak value). During the DEP process, the electrode impedance was continuously monitored using microscope (model CN15-T31, Nippon Optical works Co., Ltd, Japan). After a desired time period, the DEP process was stopped and ultrapure was gently dried out at room temperature to prepare the microelectrode retaining MWNTs as a gas sensor. In the gas experiments, $\mathrm{NH}_{3}$ was employed as a sample gas. The same microelectrode chamber as used in the DEP fabrication process was employed also for the gas sensing experiments. The chamber was firstly filled with air and then $\mathrm{NH}_{3}$ was introduced. After each sensing cycle, the chamber was flushed with air. The sensor impedance was continuously measured at room temperature using the universal meter (model UT805A, yuanhengtong Co., Ltd, China).

\section{Results and discussion}

\section{Dielectrophoretic manipulation of the raw MWNTs and MWCNTs-x}

Microscope images of raw MWNTs and MWCNTs-x trapped on the microelectrode are shown in Fig.1.The trapped MWNTs seemed to be aligned along the electric field line and bridged over the electrode tips.
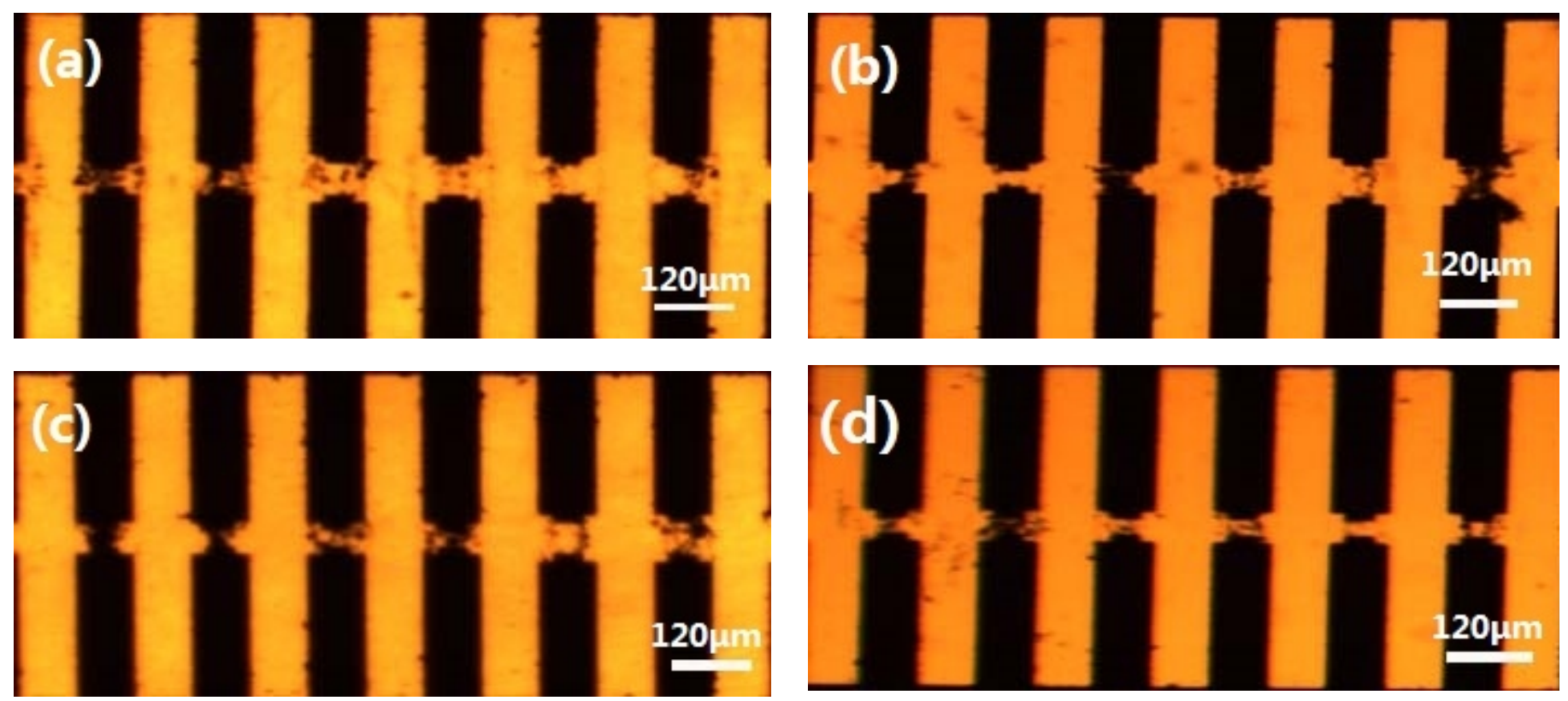

Fig. 1. Microscope images of MWNTs trapped onto microelectrode by positive DEP: (a) raw MWNTs; (b) MWNTs-OH; (c) MWNTs-COOH; (d) MWNTs-NH 2. 


\section{Gas sensing performance}

The dynamic responses of MWNTs gas sensors exposure to $\mathrm{NH}_{3}$ with concentrations from 10 ppm to 100 ppm are shown in Fig.2.
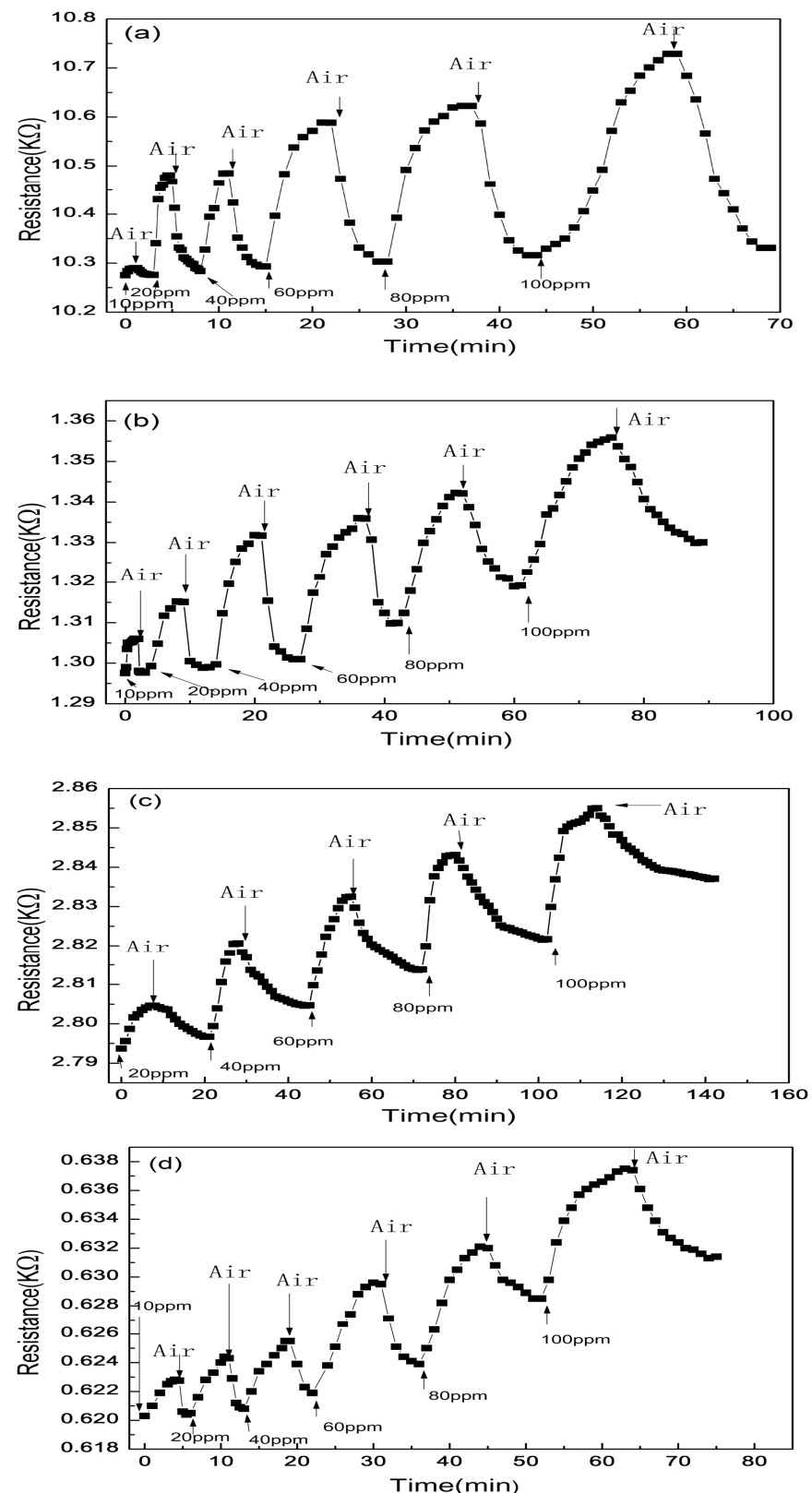

Fig.2. Dynamic response of MWNTs to different concentrations of $\mathrm{NH}_{3}$ : (a) raw MWNTs; (b) MWNTs-OH; (c) MWNTs-COOH; (d) MWNTs- $\mathrm{NH}_{2}$.

In order to examine effects of the MWNTs modified with different functional groups $(-\mathrm{OH}$, $-\mathrm{COOH},-\mathrm{NH}_{2}$ ), four sensors were fabricated. The sensitivity $(S)$ of the sensor is defined as $S=\Delta R / R_{0} \times 100 \%$, where $\Delta R$ is the resistance change after $\mathrm{NH}_{3}$ exposure and $R_{0}$ is the initial resistance. When the sensor was exposed to $\mathrm{NH}_{3}$, the resistance increased for all MWNTs sensors. The resistance increase of MWNTs by $\mathrm{NH}_{3}$ exposure suggested that MWNTs, MWNTs-OH, MWNTs-COOH and MWNTs- $\mathrm{NH}_{2}$ were p-type semiconductors. The charge transfer between $\mathrm{NH}_{3}$ and MWNTs results in a reduced hole density in MWNTs, and thus an increased resistance ${ }^{[13]}$. It was found that the response time, recovery time and sensitivity of gas sensors towards $\mathrm{NH}_{3}$ were increased with the increase of $\mathrm{NH}_{3}$ concentrations. When the $\mathrm{NH}_{3}$ was replaced with air, the resistance returned to the initial value.

Fig.3. plots the sensitivity and response time of MWNTs and MWNTs-x gas sensors measured 
in the $\mathrm{NH}_{3}$ concentration rang of $10-100 \mathrm{ppm}$. We demonstrated that functionalizing MWNTs does not change the adsorption properties of $\mathrm{NH}_{3}$ on MWNTs. Raw MWNTs sensor showed a $1.14 \%$ reversible resistance change to $10 \mathrm{ppm} \mathrm{NH}_{3}$ with a response time of $30 \mathrm{~s}$. Higher sensitivity of raw MWNTs is observed because of the lager surface area and small diameter that allows faster diffusion of gas into the MWNTs. The smaller diameters give a shorter response time because diffusion into small-diameter cylinders is fast. The normalized sensitivity of the MWNTs sensor is five times higher than that of the MWNTs-COOH sensor.

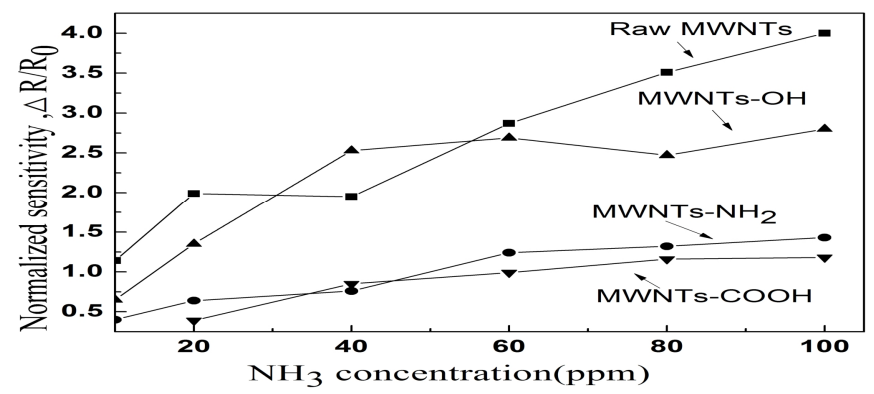

Fig.3. The sensitivity comparison of raw MWCNTs, MWCNTs-OH, MWCNTs-COOH, MWCNTs-NH 2 gas sensors.

\section{Conclusions}

A MWNTs-based $\mathrm{NH}_{3}$ sensor was successfully manipulated by positive DEP on the chromium-silver-gold microelectrode chips. The gas sensing performances of the fabricated gas sensors towards different concentrations of $\mathrm{NH}_{3}$ were tested at room temperature. It was found that the response time, recovery time and sensitivity of gas sensors towards $\mathrm{NH}_{3}$ were increased with the increase of $\mathrm{NH}_{3}$ concentrations. We demonstrated that functionalizing MWNTs does not change the adsorption properties of $\mathrm{NH}_{3}$ on MWNTs. Raw MWNTs sensor showed a $1.14 \%$ reversible resistance change to $10 \mathrm{ppm} \mathrm{NH}_{3}$ with a response time of $30 \mathrm{~s}$.

Acknowledgements: The project was supported by the National Natural Science Foundation of China (51206009), China Scholarship Council (201408110015) and Beijing Higher Education Young Elite Teacher Project (YETP1663).

\section{References}

[1] Llobet E. 2013. Gas sensors using carbon nanomaterials: A review. Sensors and Actuators B: Chemical 179:32-45.

[2] Cheng, Y. W.; Yang, Z.; Wei, H.; Wang, Y. Y.; Wei, L. M.; Zhang, Y. F. 2010 Progress in Carbon Nanotube Gas Sensor Research. Acta Phys. -Chim. Sin 26: 3127.

[3] Li W, Jung H, Hoa ND, Kim D, Hong S-K, Kim H. 2010. Nanocomposite of cobalt oxide nanocrystals and single-walled carbon nanotubes for a gas sensor application. Sensors and Actuators B: Chemical 150:160-166.

[4] De Pascali G, Melisi D, Valentini M, Valentini A, Nitti MA, et al. 2014. Spray deposited carbon nanotubes for organic vapor sensors. Microelectronics Journal 45:1691-1694.

[5] Ghaddab B, Sanchez JB, Mavon C, Paillet M, Parret R, et al. 2012. Detection of $\mathrm{O}_{3}$ and $\mathrm{NH}_{3}$ using hybrid tin dioxide/carbon nanotubes sensors: Influence of materials and processing on sensor's sensitivity. Sensors and Actuators B: Chemical 170:67-74.

[6] Suehiro J, Zhou G, Hara M. 2005. Detection of partial discharge in SF6 gas using a carbon nanotube-based gas sensor. Sensors and Actuators B: Chemical 105:164-169.

[7] Obirai JC, Hunter G, Dutta PK. 2008. Multi-walled carbon nanotubes as high temperature carbon monoxide sensors. Sensors and Actuators B: Chemical 134:640-646.

[8] Penza M, Rossi R, Alvisi M, Cassano G, Signore MA, et al. 2008. Pt- and Pd-nanoclusters functionalized carbon nanotubes networked films for sub-ppm gas sensors. Sensors and Actuators B: Chemical 135:289-297. 
[9] Hajihashemi R, Rashidi AM, Alaie M, Mohammadzadeh R, Izadi N. 2014. The study of structural properties of carbon nanotubes decorated with $\mathrm{NiFe} 2 \mathrm{O} 4$ nanoparticles and application of nano-composite thin film as H2S gas sensor. Materials science \& engineering. C, Materials for biological applications 44:417-421.

[10]Jung D, Han M, Lee GS. 2014. Gas sensor using a multi-walled carbon nanotube sheet to detect hydrogen molecules. Sensors and Actuators A: Physical 211:51-54.

[11]Dhall S, Jaggi N, Nathawat R. 2013. Functionalized multiwalled carbon nanotubes based hydrogen gas sensor. Sensors and Actuators A: Physical 201:321-327.

[12] Penza M, Cassano G, Aversa P, Antolini F, Cusano A, Consales M, Giordano M, Nicolais L. 2005. Carbon nznotubes-coated mulita-transducing sensors for VOCs detection. Sensors and Actuators B: Chemical 111-112:171-180.

[13] He L, Jia Y, Meng F, Li M, Liu J. 2009. Gas sensors for ammonia detection based on polyaniline-coated multi-wall carbon nanotubes. Materials Science and Engineering: B $163: 76-81$. 